\title{
Application of transfer matrix and transfer function analysis to grating-type dielectric laser accelerators: Ponderomotive focusing of electrons
}

\author{
Andrzej Szczepkowicz \\ Institute of Experimental Physics, University of Wroclaw, Plac Maksa Borna 9, 50-204 Wroclaw, Poland
} (Received 25 May 2017; published 31 August 2017)

\begin{abstract}
The question of suitability of transfer matrix description of electrons traversing grating-type dielectric laser acceleration (DLA) structures is addressed. It is shown that although matrix considerations lead to interesting insights, the basic transfer properties of DLA cells cannot be described by a matrix. A more general notion of a transfer function is shown to be a simple and useful tool for formulating problems of particle dynamics in DLA. As an example, a focusing structure is proposed which works simultaneously for all electron phases.
\end{abstract}

DOI: 10.1103/PhysRevAccelBeams.20.081302

\section{INTRODUCTION}

Several recent proof-of-principle experiments demonstrate the possibility of accelerating electrons in a laserdriven dielectric structure [1,2]. One class of such dielectric laser accelerator (DLA) structures is the grating-type structure, in which a unit cell is iterated in one dimension, as in the recently developed single grating, dual-grating, and dual pillar structures [2]. On the basis of these successful experiments, compact laser driven accelerators are envisioned (see for example Fig. 4 in Ref. [3]). A working device will require, in addition to acceleration, beam focusing, and possibly beam diagnostics sections and feedback beam steering. To design a complete DLA beam line, a mathematical description of electron trajectory throughout the whole device is necessary. For conventional radio-frequency (rf) accelerators, several mathematical tools were developed over the years to effectively describe the single particle and beam trajectories [4-7]. One such tool is the transfer matrix; it is used to describe the particle transfer properties of the various building blocks of a beam line. For grating-type DLAs, the natural building block is the unit cell of the grating [8]. Here, interesting questions arise: what are the particle transfer properties of a DLA unit cell, and can they be described by a matrix? This problem has been partially addressed in Ken Soong's Ph.D. thesis [9], where the transfer matrix of a unit cell of a doublegrating accelerator structure is calculated. In this pioneering work the adequacy of linear approximation is not discussed, and a 25-attosecond electron bunch is assumed, with length less than $1 \%$ of the grating period, evading the

Published by the American Physical Society under the terms of the Creative Commons Attribution 4.0 International license. Further distribution of this work must maintain attribution to the author(s) and the published article's title, journal citation, and DOI. problem of distribution of phases. The purpose of the present work is to pursue further this interesting idea.

\section{THE TRANSFER MATRIX FORMALISM}

In conventional rf accelerators particle motion is described relative to a reference trajectory $[4,10]$. The reference trajectory defines a coordinate system which is in general curvilinear, with the distance along the trajectory described by coordinate $S$ (following the notation in Ref. [10]), and with orthogonal coordinates $x, y$ describing the particle position in the transverse plane. The particle on the reference trajectory has reference energy $\mathcal{E}_{0}$ (corresponding to reference momentum $p_{0}$ ). The relative position of electrons on the reference trajectory with respect to the beam center is measured by $s$. The electron location in the six-dimensional phase space comoving with the electron beam is characterized by the vector $\vec{X}=\left(x, x^{\prime}, y, y^{\prime}, s, \eta\right)^{\mathrm{T}}$ [10], where $x^{\prime}=d x / d S$ and $y^{\prime}=d y / d S$ are the small angles of deflection from the reference trajectory, and $\eta=\Delta \mathcal{E} / \mathcal{E}_{0}$ is the relative energy deviation (other authors [4,5] use relative momentum deviation $\delta=\Delta p / p_{0}$ instead of $\eta$; in the ultrarelativistic limit $\eta=\delta$ ). Note that all coordinates of $\vec{X}$ are small and the reference particle is described by $\vec{X}=(0,0,0,0,0,0)^{\mathrm{T}}$.

In conventional rf accelerators, basic properties of a beam line section can be described by first-order beam transport optics [4], using linear approximation:

$$
\vec{X}_{2}=\mathcal{R} \vec{X}_{1},
$$

where $\vec{X}_{1}$ describes a particle at the entrance of the section, $\vec{X}_{2}$-at the exit of the section, and $\mathcal{R}$ is a linear transfer function, which is represented by a $6 \times 6$ transfer matrix. Phenomena not captured by this approximation can be described by second-order optics [4] or by detailed numerical particle tracing. 


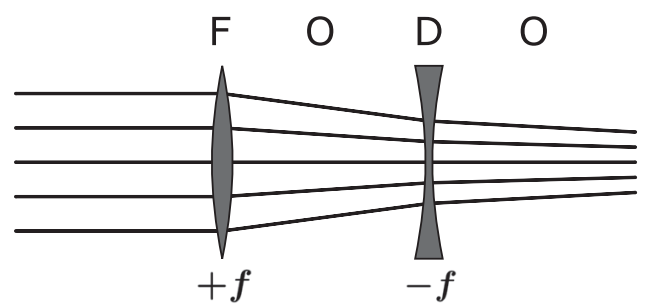

FIG. 1. The principle of FODO focusing. The best known realization of this principle is the alternating gradient focusing used in conventional rf accelerators.

Often in the literature a reduced form of the $\mathcal{R}$ matrix is used [4-7], where, as a starting point of the analysis, chromatic effects are neglected $(\delta=0, \eta=0)$, and only the $\left(x, x^{\prime}\right)$ phase plane is considered:

$$
\left(\begin{array}{l}
x_{2} \\
x_{2}^{\prime}
\end{array}\right)=\left(\begin{array}{ll}
R_{11} & R_{12} \\
R_{21} & R_{22}
\end{array}\right)\left(\begin{array}{c}
x_{1} \\
x_{1}^{\prime}
\end{array}\right) .
$$

In the context of classical optics, such a formulation is called ray transfer matrix analysis (or ABCD matrix analysis) and is used to describe the propagation of light rays and Gaussian beams in the paraxial approximation [11]. Beam transfer through a thin lens of focal length $f$ is described by the matrix

$$
\mathcal{F}=\left(\begin{array}{cc}
1 & 0 \\
-1 / f & 1
\end{array}\right)
$$

( $1 / f$ is called optical power or focusing power). A free drift region of length $s$ with no optical elements is described by the transfer matrix

$$
\mathcal{O}=\left(\begin{array}{ll}
1 & s \\
0 & 1
\end{array}\right)
$$

One of the common building blocks used in the design of rf accelerator beam lines is the FODO array (focusing-driftdefocusing-drift) [4-7], which has an overall focusing effect, see Fig. 1. The transfer function of the FODO array is the mathematical composition of the transfer functions of its four building blocks. Composition of linear functions is equivalent to matrix multiplication:

$$
\begin{aligned}
\mathcal{R}_{\mathrm{FODO}}= & \mathcal{O D O} \mathcal{F}=\left(\begin{array}{ll}
1 & s \\
0 & 1
\end{array}\right)\left(\begin{array}{cc}
1 & 0 \\
-1 /(-f) & 1
\end{array}\right) \\
& \times\left(\begin{array}{ll}
1 & s \\
0 & 1
\end{array}\right)\left(\begin{array}{cc}
1 & 0 \\
-1 / f & 1
\end{array}\right) \\
= & \left(\begin{array}{cc}
\frac{f^{2}-f s-s^{2}}{f^{2}} & \frac{s(2 f+s)}{f} \\
-\frac{s}{f^{2}} & \frac{f+s}{f}
\end{array}\right) .
\end{aligned}
$$

The focusing power of the FODO structure is

$$
\frac{1}{f_{\mathrm{FODO}}}=\frac{R_{21}}{R_{11}}=\frac{(1 / f)^{2} s}{1-s / f-(s / f)^{2}} .
$$

If the focal length is much larger than the length of the drift region, $f \gg s$, the expression simplifies to

$$
\frac{1}{f_{\mathrm{FODO}}} \approx\left(\frac{1}{f}\right)^{2} s
$$

so in the thin and weak lens approximation, the focusing power of the FODO structure is proportional to the square of the constituent lens' focusing power. This result will be recalled in Sec. V.

\section{ELECTRON TRANSFER ANALYSIS FOR GRATING-TYPE DIELECTRIC LASER ACCELERATORS}

Let us try to develop a methodology, similar to the one outlined in Sec. II, to describe electron transfer through a grating-type DLA. In this context it is natural to use a Cartesian coordinate system, see Fig. 2. The structure is driven by laser pulses from the direction perpendicular to the electron beam. It is assumed here that the structure exhibits no large-scale resonances such as guided-mode
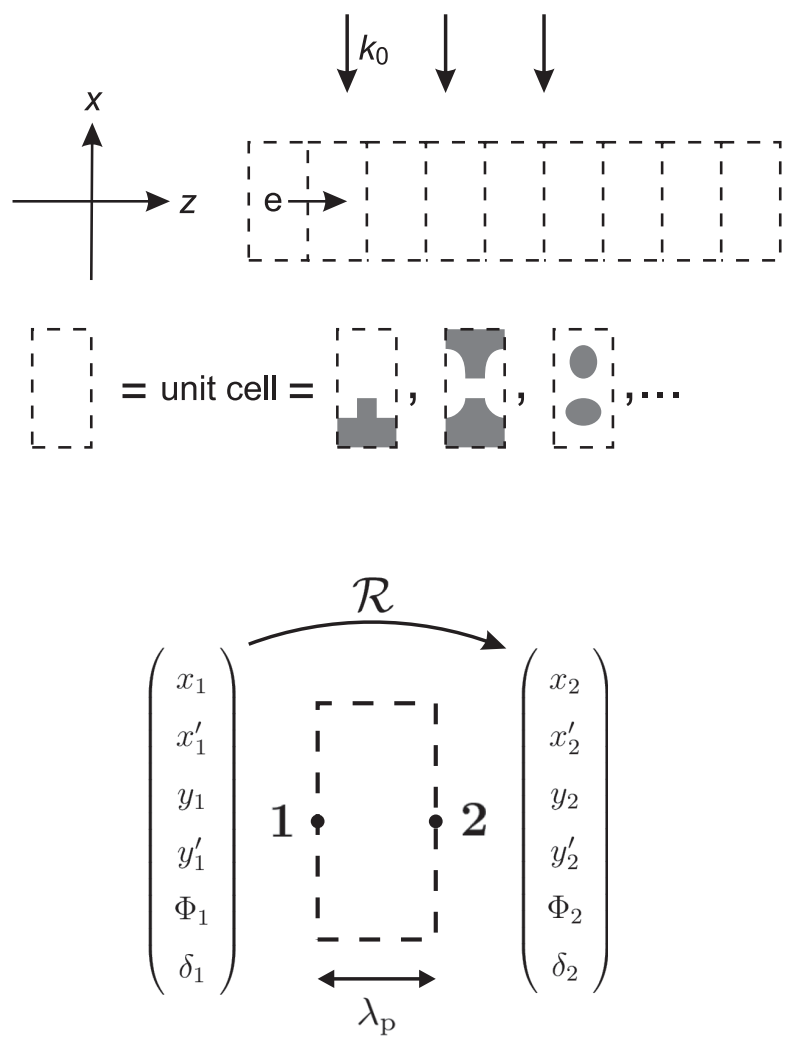

FIG. 2. A segment of a grating-type DLA. The unit cell is iterated in one dimension along the electron beam. 
resonances [12], so that the filling times are shorter than the laser pulse length. With this assumption stationary (timeharmonic) calculation of the electromagnetic field is appropriate, and one can obtain a realistic time-dependent field by multiplying the stationary result by the laser pulse envelope. This scaling of the result is not carried out here, as it would not affect the conclusions. Let $\left(\tilde{E}_{x}, \tilde{E}_{y}, \tilde{E}_{z}, \tilde{B}_{x}, \tilde{B}_{y}, \tilde{B}_{z}\right)$ represent the stationary solution of the electromagnetic field in a given structure; $E_{x}(x, y, z, t)=\Re\left[\tilde{E}_{x}(x, y, z) e^{i \omega_{0} t}\right]$, etc. For a start, assume that electron velocity is perfectly aligned with $\hat{z}$. If the velocity $\beta_{0} c$ is tuned perfectly to the grating period $\lambda_{\mathrm{p}}$ and laser wavelength $\lambda_{0}$, then $\beta_{0}=\lambda_{\mathrm{p}} / \lambda_{0}=k_{0} / k_{\mathrm{p}}$, assuming that the DLA is operated at first spatial harmonic [13]. Let us call $\beta_{0} c$ the reference velocity, corresponding to the reference momentum

$$
p_{0}=\frac{m \beta_{0} c}{\sqrt{1-\beta_{0}^{2}}}
$$

Let $\delta$ denote the electron's relative deviation from the reference momentum:

$$
\delta=\frac{p-p_{0}}{p_{0}} .
$$

Electron position in the transverse plane is described by $(x, y)$, and the slope of the trajectory is described by

$$
\left(x^{\prime}, y^{\prime}\right)=\left(\frac{d x}{d z}, \frac{d y}{d z}\right)
$$

In a radio-frequency accelerator, particle bunch duration $\tau$ is $\sim 3$ orders of magnitude smaller than the period of the driving electromagnetic wave: $\tau \ll T_{0} \approx 10^{-10} \mathrm{~s}$. In contrast to this, in DLA, the inequality is reversed: $\tau \gg T_{0} \approx 10^{-14} \mathrm{~s}$, due to limitations of the present day electron sources (see eg. [14]); another limiting factor is the space charge force [13]. As a result, in DLA electrons in a bunch populate all phases. In the context of grating-type DLAs, phase appears more important than longitudinal position of the electron along the grating, so it will be convenient to use a parameter $\Phi$ (radians) instead of $S$ (meters) to describe electron's longitudinal degree of freedom. Let us define $\Phi_{1}$ of an electron as the phase of the electromagnetic field at the moment $t_{1}$ when the electron enters the unit cell of the grating:

$$
\Phi_{1}=\omega_{0} t_{1} .
$$

For an electron with $x^{\prime}=0, y^{\prime}=0$ and reference momentum $p=p_{0}$, traversing the unit cell from $z=z_{1}$ to $z=z_{1}+\lambda_{p}=z_{2}$, the phase increases from $\Phi_{1}$ to $\Phi_{1}+2 \pi=\Phi_{2}$. Note that in contrast to $x, x^{\prime}, y, y^{\prime}$ and $\delta$, the parameter $\Phi$ is not small; it is analogous to the parameter $S$ defined in Sec. II, not the small parameter $s$. A parameter analogous to $s$ would be $\phi=\Phi_{2}-\Phi_{1}-2 \pi$.

The set of parameters $\left(x_{1}, x_{1}^{\prime}, y_{1}, y_{1}^{\prime}, \Phi_{1}, \delta_{1}\right)$ fully describes the classical motion state of a particle at the entrance of the unit cell. Therefore there exists a transfer function $\mathcal{R}$, such that

$$
X_{2}=\mathcal{R} X_{1} \text {, }
$$

where $X_{1}=\left(x_{1}, x_{1}^{\prime}, y_{1}, y_{1}^{\prime}, \Phi_{1}, \delta_{1}\right)^{\mathrm{T}}$ are the parameters of the electron at the entrance of the unit cell, and $X_{2}=\left(x_{2}, x_{2}^{\prime}, y_{2}, y_{2}^{\prime}, \Phi_{2}, \delta_{2}\right)^{\mathrm{T}}$ are the parameters of the electron at the exit of the unit cell, see Fig. 2. A matrixlike notation is used here, where one-column matrix $X_{2}$ is the result of operator $\mathcal{R}$ acting on one-column matrix $X_{1}$.

Using (13), the properties of $\mathcal{R}$ can be studied numerically (particle tracing) even without explicit formulas for $\mathcal{R}$, by specifying sets of example parameters $\left\{X_{1}\right\}$ and calculating corresponding sets of $\left\{X_{2}\right\}$. Explicit formulas for $\mathcal{R}$ are given in Appendix A; these formulas were used in subsequent analyses.

\section{EXAMPLE TRANSFER FUNCTION ANALYSIS: A DOUBLE COLUMN STRUCTURE}

Let us now apply the concepts of Sec. III to a specific example of a grating-type DLA: the double-column structure described in Ref. [15]. Figure 3 shows the unit cell. The columns are long enough so that the system can be described in two dimensions $(z, x)$, assuming infinite column extension in the $\hat{y}$ direction [15]. The $y$ coordinate is not significant and will be set to 0 . Let us study some of the properties of the transfer function of the unit cell. First, the electromagnetic field is calculated using the finite element method. Then the transfer function is applied

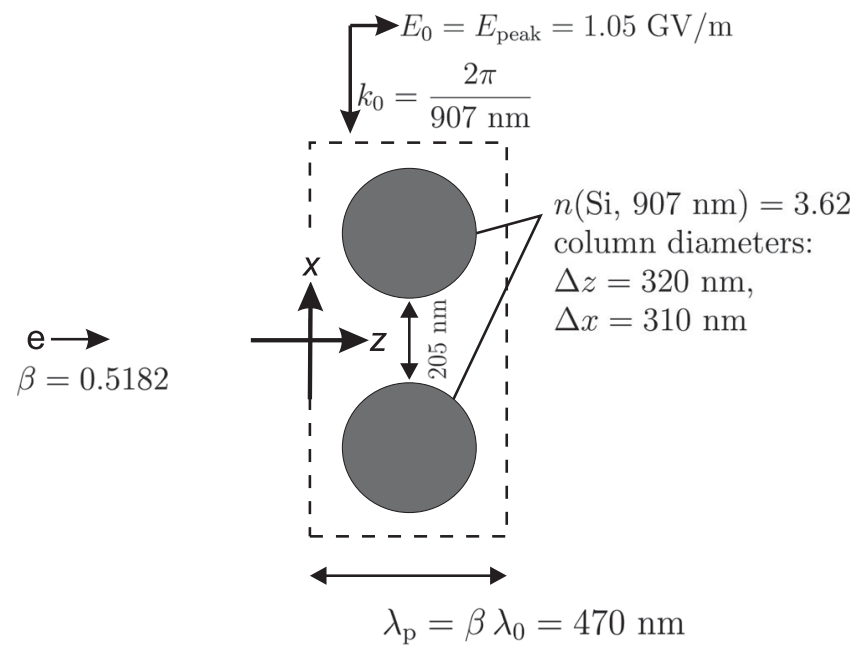

FIG. 3. Example parameters used for calculation, based on Ref. [15]. 

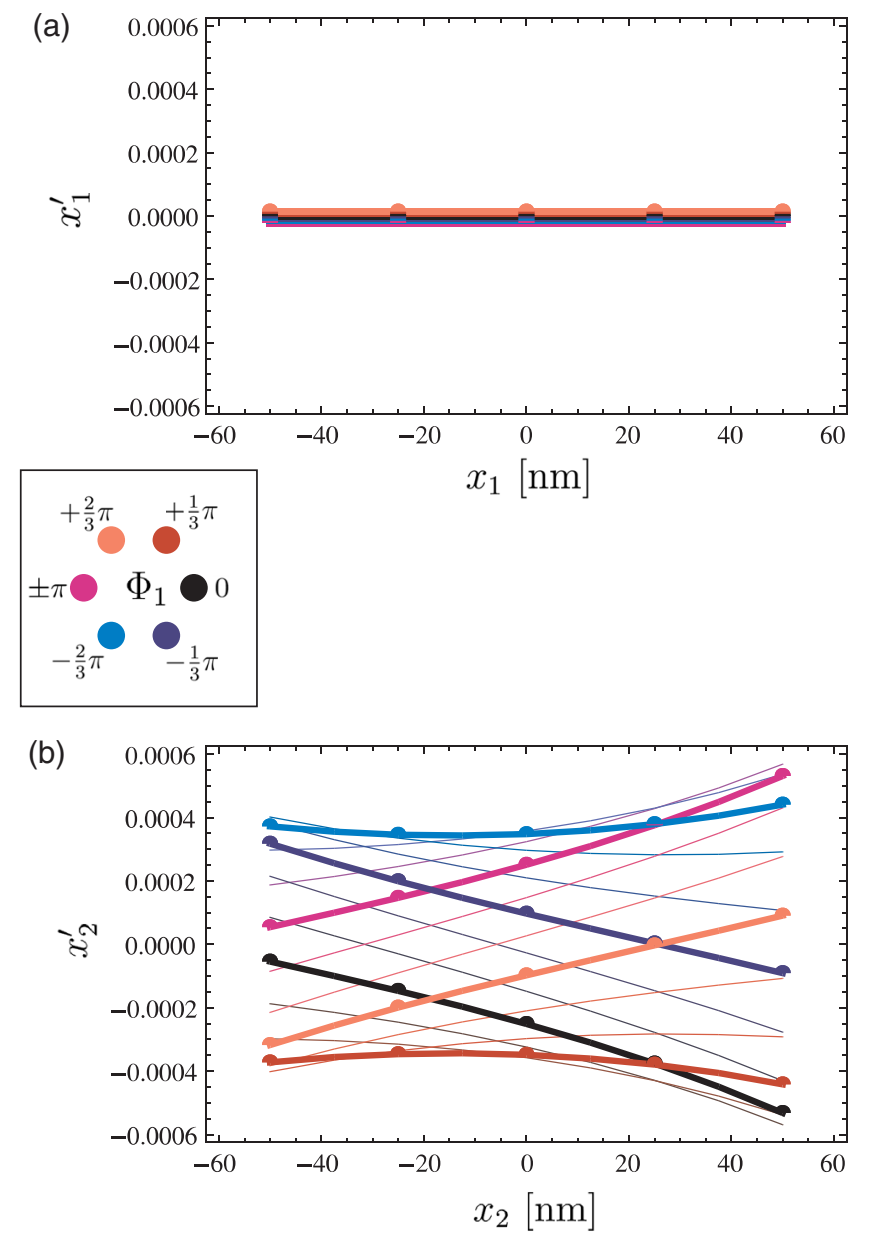

FIG. 4. The properties of the transfer function for the unit cell shown in Fig. 3. (a) Assumed $\left(x_{1}, x_{1}^{\prime}\right)$ at the entrance of the cell. (b) Calculated $\left(x_{2}, x_{2}^{\prime}\right)$ at the exit of the cell. The result depends on the initial phase $\Phi_{1}$ (color coded). The thicker lines correspond to six selected phases: $-\pi,-\frac{2}{3} \pi,-\frac{1}{3} \pi, 0, \frac{1}{3} \pi, \frac{2}{3} \pi$.

to sample input parameters using equations given in Appendix A. Suppose the incoming electrons are parallel to the $\hat{z}$ direction: $x_{1}^{\prime}=0, y_{1}^{\prime}=0$, and have reference momentum: $p=p_{0}$, so that $\delta_{1}=0$. For a start let us choose an initial phase $\Phi_{1}=0$ and a set of initial electron positions: $\left\{x_{1, i}\right\}=\{-50 \mathrm{~nm},-25 \mathrm{~nm}, 0 \mathrm{~nm},+25 \mathrm{~nm},+50 \mathrm{~nm}\}$. The result of applying $\mathcal{R}$ to $X_{1, i}=\left(x_{1, i}, 0,0,0, \Phi_{1}, 0\right)^{\mathrm{T}}$ is $X_{2, i}=\left(x_{2, i}, x_{2, i}^{\prime}, 0,0, \Phi_{2, i}, \delta_{2, i}\right)^{\mathrm{T}}$. With this set of calculated parameters various plots are possible. An example is shown in Fig. 4, where in (a) pairs $\left(x_{1}, x_{1}^{\prime}\right)$ are plotted, while (b) shows $\left(x_{2}, x_{2}^{\prime}\right)$ pairs (black curve). Subsequently, another initial phase $\Phi_{1}$ is selected and the procedure is repeated, with results plotted in different colors in the same figure.

The main question that motivated the described investigations was: is transfer matrix description suitable for grating-type DLA structures? The answer follows easily from Fig. 4. The transfer function does not in general transform $(0,0,0,0,0,0)^{\mathrm{T}}$ into $(0,0,0,0,0,0)^{\mathrm{T}}$, so it is not
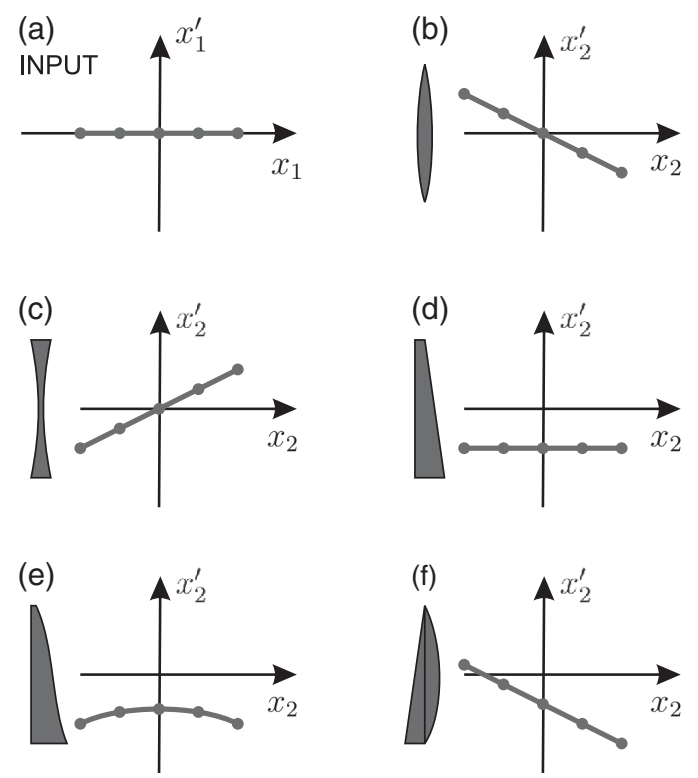

FIG. 5. The transfer properties of simple optical elements: (a) The assumed set of input pairs $\left(x_{1}, x_{1}^{\prime}\right)$, representing incident rays with various positions and zero slopes. (b)-(f) Calculated $\left(x_{2}, x_{2}^{\prime}\right)$ at the exit of (b) converging lens, (c) diverging lens, (d) linear prism, (e) nonlinear prism, (f) converging lens with off-axis focus. Linear transfer functions describe (a)-(c), for (d), (f) an affine function is needed, while for (e) a nonlinear transfer function must be used.

a linear function and it cannot be described by a matrix. Even if $\Phi$ is excluded from the set of transformed parameters and one looks for a reduced $\mathcal{R}^{\prime}$ operating in the $\left(x, x^{\prime}\right)$ space, Fig. 4 shows that in general $\mathcal{R}^{\prime}(0,0)^{\mathrm{T}} \neq(0,0)^{\mathrm{T}}$, so the matrix description is not possible. For example, an electron entering the unit cell with phase $\Phi_{1}=-\frac{2}{3} \pi$ and zero slope leaves the cell with nonzero slope $x_{2}^{\prime} \approx 0.0004$. What is more, neither $\mathcal{R}$ nor $\mathcal{R}^{\prime}$ belong to the wider class of affine transforms (linearity with an offset), because the plots in Fig. 4(b) are not rectilinear. Here and in subsequent considerations chromatic effects are neglected: $\delta_{1}=0$ is assumed.

Let us compare the calculated transfer properties with optical transfer properties of glass solids, Fig. 5. As can be seen from comparison of Figs. 4 and 5, the accelerator unit cell, depending on the incoming electron's phase $\Phi_{1}$, acts as a converging lens for $\Phi_{1} \in\left(-\frac{1}{3} \pi, 0\right)$, a diverging lens for the opposite phase $\Phi_{1} \in\left(+\frac{2}{3} \pi,+\pi\right)$, an upward-deflecting nonlinear prism (larger deflection for larger $\left|x_{1}\right|$ ) for $\Phi_{1} \approx-\frac{2}{3} \pi$, and a downward-deflecting nonlinear prism for $\Phi_{1} \approx+\frac{1}{3} \pi$.

\section{PONDEROMOTIVE FOCUSING IN GRATING- TYPE DIELECTRIC LASER ACCELERATORS}

In conventional accelerators the primary method of focusing is alternating gradient focusing (also called 


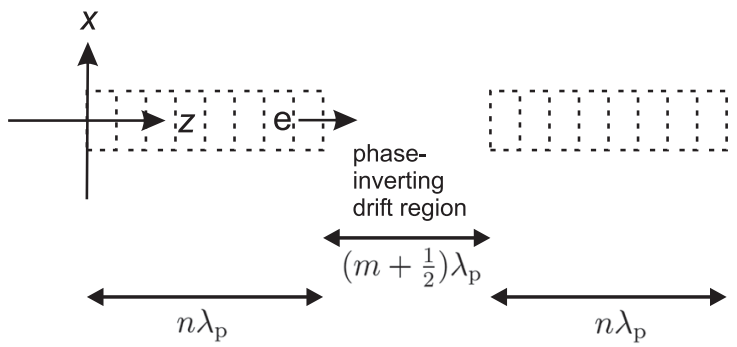

FIG. 6. A DLA accelerator segment analogous to a FODO structure.

strong focusing), where lensing quadrupole magnets generate field gradient $\partial B / \partial x, \partial B / \partial y$, and are arranged along the beam direction $z$ with alternating polarity. This is an implementation of the FODO focusing principle described in Sec. II. Alternating gradient focusing will be used in planned hybrid accelerator experiments, where a rf beam line will be matched to grating-type DLAs [16,17]. Of course, the ultimate goal is to develop compact accelerators employing optical-frequency focusing. At present, laser focusing is in the early development stage, with conceptual and simulation work under way [18-20], and a first proofof-principle experiment with parabolic grating [21]. One major problem with focusing in DLA is the same as with acceleration: as yet the phase of electrons in not controlled experimentally, and a shift of phase by $\pi$ reverses the force of the electromagnetic field on the particle and turns focusing into defocusing, so only a fraction of electrons is focused. Is it possible to focus electrons with different phases $\Phi$ at the same time?

An interesting property of a FODO structure is that it keeps its focusing properties if the forces are reversed: both $\mathcal{O D O} \mathcal{F}$ and $\mathcal{O F O D}$ are focusing transformations. Suppose an electron enters a DLA structure shown in Fig. 6, and the unit cell has similar transfer properties as in Fig. 4. The transfer function of the whole structure is

$$
\mathcal{R}_{\text {tot }}=\mathcal{O}_{m+1 / 2} \mathcal{R}^{n} \mathcal{O}_{m+1 / 2} \mathcal{R}^{n},
$$

where again matrixlike notation is used, with multiplication representing mathematical composition of functions, $\mathcal{R}^{n}$ denoting the composition of $n$ single cell transfer functions $\mathcal{R}$, and $\mathcal{O}_{m+1 / 2}$ denoting the linear drift operator (4) for $s=\left(m+\frac{1}{2}\right) \lambda_{\mathrm{p}}$. If, for an electron with phase $\Phi, \mathcal{R}$ has focusing properties, then $\mathcal{R}^{n}$ is also focusing (for $n$ small enough so that dephasing [21] is not significant). The drift section $\mathcal{O}_{m+1 / 2}$ advances the electron phase by $2 \pi m+\pi$, so the second $\mathcal{R}^{n}$ section is defocusing — just like in a FODO structure. If another electron enters the same structure with phase $\Phi+\pi$, the structure acts on it as DOFO. For both electrons the structure acts as a converging lens. Consider now an electron with such a phase $\Phi^{\prime}$ that the unit cell acts as a nonlinear upward-deflecting prism. Now the whole structure cannot be classified as FODO. After traversing the
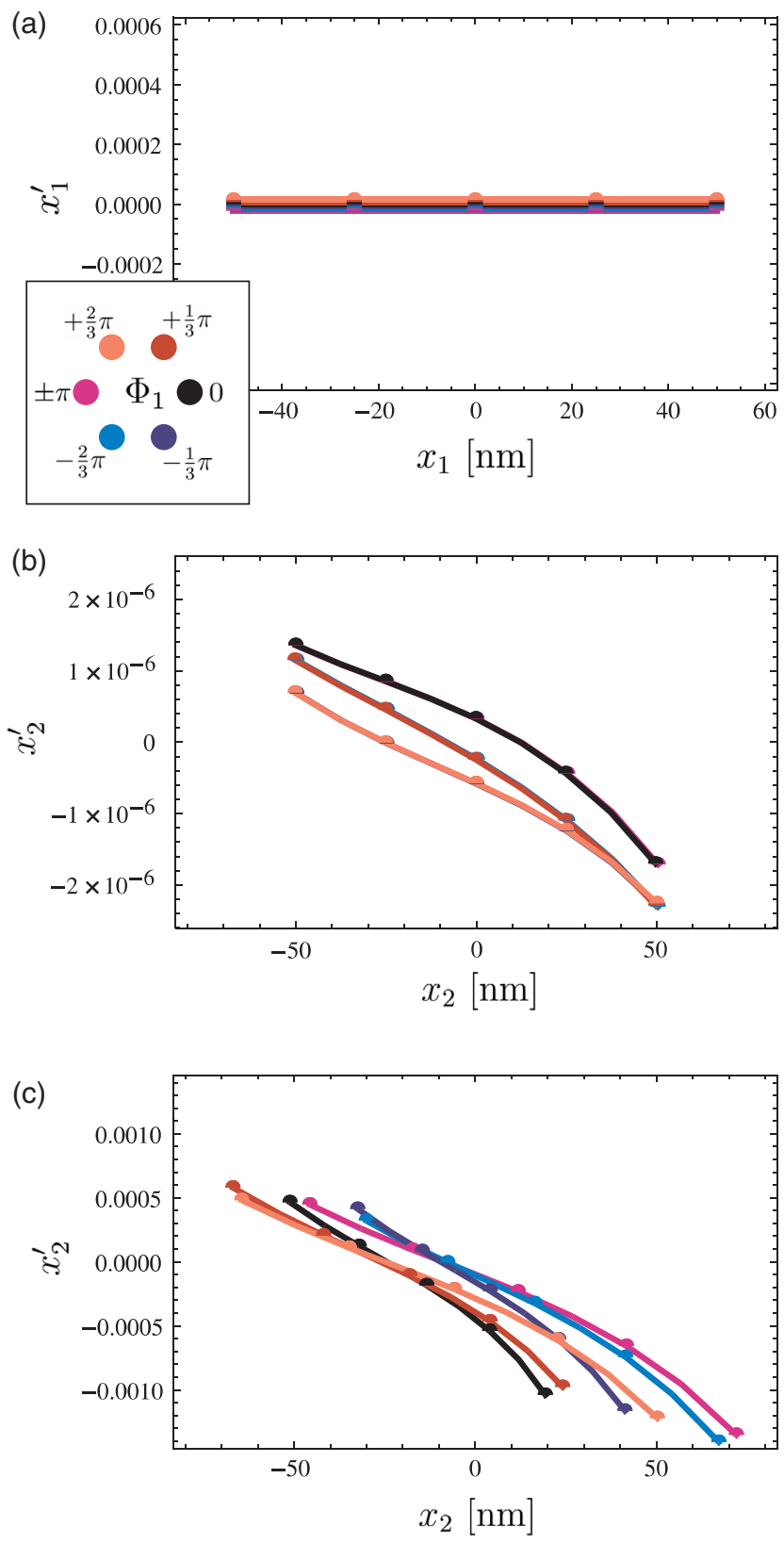

FIG. 7. The focusing properties of the accelerator segment shown in Fig. 6, calculated for the unit cell shown in Fig. 3 using Eq. (A1). (a) Assumed $\left(x_{1}, x_{1}^{\prime}\right)$ at the entrance of the segment: a parallel beam. (b) Calculated $\left(x_{2}, x_{2}^{\prime}\right)$ at the exit of the segment, for $n=1$ and $m=0$. (c) Calculated $\left(x_{2}, x_{2}^{\prime}\right)$ at the exit of the segment, for $n=8$ and $m=5$.

first $\mathcal{R}^{n}$ section, the electron is deflected upwards, $\mathcal{O}_{m+1 / 2}$ reverses the phase, and in the second $\mathcal{R}^{n}$ section the electron is deflected downwards. However, because the "prism" $\mathcal{R}^{n}$ is nonlinear, its action is stronger away from the $x=0$ line and the overall effect of $\mathcal{R}_{\text {tot }}$ is again a converging lens. A similar argument applies to an electron entering the structure with $\Phi^{\prime}+\pi$ phase. This reasoning, based on $\left(x, x^{\prime}\right)$ plots, is purely geometric, but a chromatic 
effect $(\delta \neq 0)$ also plays a role in focusing, as shown in Appendix C.

The phase-independent focusing effect of $\mathcal{R}_{\text {tot }}$ is shown in Fig. 7. This structure is a converging lens that exhibits both geometric and "phase" aberrations. The focal lengths for the structure $\mathcal{O}_{1 / 2} \mathcal{R} \mathcal{O}_{1 / 2} \mathcal{R}$, as shown in Fig. 7(b), lie in the range $30-35 \mathrm{~mm}$, so the focusing effect is very weak. The focal lengths for the structure $\mathcal{O}_{5+1 / 2} \mathcal{R}^{8} \mathcal{O}_{5+1 / 2} \mathcal{R}^{8}$, as shown in Fig. 7(c), lie in the range $48-70 \mu \mathrm{m}$, so the focusing effect is 3 orders of magnitude stronger. This shows that grouping of the unit cells is critical (see also Ref. [22]). The effect of grouping is even stronger than for a thin lens FODO structure described by Eq. (8) (see also Appendix D). However, grouping increases the chance of electron collision with the dielectric. It is likely that the geometry of the unit cell (Fig. 3) could be optimized for better transfer and focusing performance, but this is left for future work. Also, in the presented approach boundary field effects were neglected. This is justified for large structures like $\mathcal{O}_{5+1 / 2} \mathcal{R}^{8} \mathcal{O}_{5+1 / 2} \mathcal{R}^{8}$, but the calculation of $\mathcal{O}_{1 / 2} \mathcal{R O}_{1 / 2} \mathcal{R}$ may be inaccurate. Boundary field effects can be handled with the transfer function approach by introducing intermediate boundary cells $\mathcal{B}_{ \pm}$, as shown in Fig. 8. In this approach, the transfer function $\mathcal{O}_{5+1 / 2} \mathcal{R}^{8} \mathcal{O}_{5+1 / 2} \mathcal{R}^{8}$ is an approximation of the more accurate $\mathcal{O}_{3+1 / 2} \mathcal{B}_{+} \mathcal{R}^{8} \mathcal{B}_{-} \mathcal{O}_{3+1 / 2} \mathcal{B}_{+} \mathcal{R}^{8} \mathcal{B}_{-}$.

The structure shown in Fig. 6, with its converging property, cannot in general (for arbitrary $\Phi$ ) be classified as FODO (see Fig. 9), but along with FODO it belongs to a wider class of focusing setups based on ponderomotive force [23,24] ([23] gives historical references). Quantitative similarities and differences between the classical ponderomotive force and focusing force of

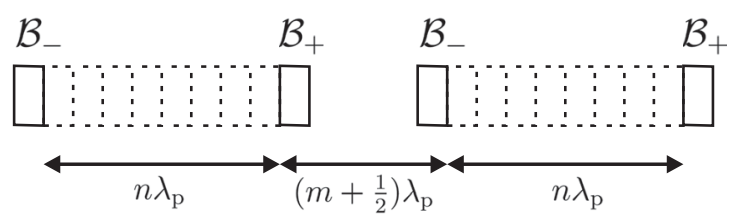

FIG. 8. Boundary field effects can be handled using boundary cells with corresponding $\mathcal{B}_{ \pm}$transfer functions.

(a)

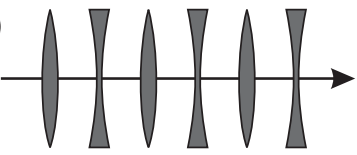

(b)

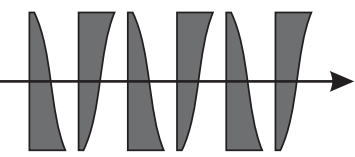

FIG. 9. Alternating lens (FODO) (a), and alternating nonlinear prisms (b), are examples of ponderomotive focusing systems. the $\mathcal{O}_{m+1 / 2} \mathcal{R}^{n} \mathcal{O}_{m+1 / 2} \mathcal{R}^{n}$ structure are discussed in Appendix D. As noted by Hartman and Rosenzweig [25], other alternating focusing schemes used in radio frequency accelerators, like radio-frequency quadrupole (RFQ) focusing [26], or alternating phase focusing $[26,27]$, are also based on ponderomotive force. In the context of DLA, a ponderomotive focusing scheme has already been studied for photonic band-gap accelerators [22]. For grating-type DLA, the idea was considered in Ref. [13] (citing [22,27]), but specific implementation was not proposed.

Ponderomotive focusing of electrons in the transverse plane is analogous to the redistribution of sand on a Chladni plate [28]. A grain of sand on a vibrating plate is subject to alternating force whose amplitude is a function of position on the plate, and diffuses towards regions of smaller amplitude, finally settling in the nodal regions. Similarly, an electron traversing a $\mathcal{O}_{m+1 / 2} \mathcal{R}^{n} \mathcal{O}_{m+1 / 2} \mathcal{R}^{n} \mathcal{O}_{m+1 / 2} \mathcal{R}^{n} \ldots$ structure with reference velocity $\beta_{0} c$ is subject to an alternating force of frequency

$$
\omega^{\prime}=\frac{\omega_{0}}{(2 n+2 m+1)}
$$

[because the spatial period of the structure is $\left.(2 n+2 m+1) \lambda_{\mathrm{p}}\right]$, and is attracted in the transverse plane towards regions of smaller force amplitude-smaller electromagnetic field. The field is stronger close to the dielectric surfaces, and for double gratinglike structures the minimum of the transverse force lies in the electron channel between the two surfaces.

\section{CONCLUSION AND OUTLOOK}

Transfer matrices are known to be useful for the description of particle motion through the segments of conventional $\mathrm{rf}$ accelerators. A similar description is proposed here for grating-type DLAs: linear transfer functions, represented by matrices, are replaced by nonlinear transfer functions; matrix multiplication is replaced by numerical function composition; these differences are hardly noticeable with a compact, matrixlike notation. The approach facilitates quantitative description of electron transfer through a DLA structure, where, in the first approximation, the transfer properties of larger units are easily determined from the transfer properties of the DLA unit cell. Hopefully this approach will make easier the conceptual and simulation work on new designs, and help in clear presentation and discussion of the properties of new DLA structures. One example of presentation of transfer properties are the $\left(x, x^{\prime}\right)$ plots, sometimes called "trace space plots" in the rf accelerator literature; such plots are already entering the DLA literature [16], and can naturally be produced with the transfer function approach described here. 
In Sec. V the transfer function approach led naturally to the idea of building a FODO-like DLA structure, which focuses electrons irrespective of the phase. The converging force in the proposed setup is yet another example of ponderomotive force. Further work is required to optimize the geometry. One approach would be to drive the structure symmetrically from two sides by employing distributed Bragg reflectors [17].

In this paper the transfer function is applied only to lensing properties of DLA structures. Of course the primary function of DLAs is to accelerate: to increase $\delta$. Here it was assumed that $\delta_{1}=0$ and $\delta_{2}$ was not analyzed. Hopefully the described formalism with its six parameters $\left(x, x^{\prime}, y, y^{\prime}, \Phi, \delta\right)$ will also be useful to describe acceleration schemes. Here a major challenge is the phase distribution of electrons, which results in only a fraction of electrons being accelerated. To address this issue, methods to compress the particle bunch are investigated [17] to obtain single-phase particles. More generally, a method is needed to redistribute the electron phases to populate several narrow $\Phi$ subsets separated by $2 \pi$. Alternatively, perhaps an accelerating scheme working for all incoming $\Phi$ could be invented. Formulation of these challenges using $\left(x, x^{\prime}, y, y^{\prime}, \Phi, \delta\right)$ may accelerate progress in this field.

\section{ACKNOWLEDGMENTS}

I am grateful to Martin Kozák, Joshua McNeur and Peter Hommelhoff for inspiring discussions during my visit in
Erlangen in May 2016. I am grateful to Wrocław Centre for Networking and Supercomputing for granting access to the Platon computing infrastructure.

\section{APPENDIX A: TRANSFER FUNCTION EQUATIONS}

The transfer function defined by Eq. (13) can be put into the following explicit form (derived in Appendix B):

$$
\begin{gathered}
x_{2}=x_{1}+x_{1}^{\prime}\left(z_{2}-z_{1}\right) \\
x_{2}^{\prime}=\frac{x_{1}^{\prime}+\frac{\Delta p_{x}}{C p_{0}\left(1+\delta_{1}\right)}}{1+\frac{\Delta p_{z}}{C p_{0}\left(1+\delta_{1}\right)}} \\
y_{2}=y_{1}+y_{1}^{\prime}\left(z_{2}-z_{1}\right) \\
y_{2}^{\prime}=\frac{y_{1}^{\prime}+\frac{\Delta p_{y}}{C p_{0}\left(1+\delta_{1}\right)}}{1+\frac{\Delta p_{z}}{C p_{0}\left(1+\delta_{1}\right)}}
\end{gathered}
$$

$$
\Phi_{2}=\Phi_{1}+k_{0} \frac{z_{2}-z_{1}}{\beta_{z}}
$$

In Eq. (A1), the following auxiliary quantities were used: $C$ is the trajectory deflection cosine $=\hat{z} \cdot \hat{v}_{1}, \beta_{z}$ is the relative longitudinal velocity, $\Delta p_{x}, \Delta p_{y}, \Delta p_{z}$ is the momentum change of the electron. The formulas for these auxiliary quantities are

$$
\begin{gathered}
C=\frac{1}{\sqrt{x_{1}^{\prime 2}+y_{1}^{\prime 2}+1}} \\
\beta_{z}=C \frac{p_{0}\left(1+\delta_{1}\right)}{\sqrt{p_{0}^{2}\left(1+\delta_{1}\right)^{2}+m^{2} c^{2}}} \\
\Delta p_{x}=\Re\left\{\frac{(-e)}{c} \int_{z_{1}}^{z_{2}}\left(\frac{1}{\beta_{z}} \tilde{E}_{x}+y_{1}^{\prime} c \tilde{B}_{z}-c \tilde{B}_{y}\right) \exp \left[i\left(\Phi_{1}+k_{0} \frac{z-z_{1}}{\beta_{z}}\right)\right] d z\right\} \\
\Delta p_{y}=\Re\left\{\frac{(-e)}{c} \int_{z_{1}}^{z_{2}}\left(\frac{1}{\beta_{z}} \tilde{E}_{y}+c \tilde{B}_{x}-x_{1}^{\prime} c \tilde{B}_{z}\right) \exp \left[i\left(\Phi_{1}+k_{0} \frac{z-z_{1}}{\beta_{z}}\right)\right] d z\right\} \\
\Delta p_{z}=\Re\left\{\frac{(-e)}{c} \int_{z_{1}}^{z_{2}}\left(\frac{1}{\beta_{z}} \tilde{E}_{z}+x_{1}^{\prime} c \tilde{B}_{y}-y_{1}^{\prime} c \tilde{B}_{x}\right) \exp \left[i\left(\Phi_{1}+k_{0} \frac{z-z_{1}}{\beta_{z}}\right)\right] d z\right\} .
\end{gathered}
$$


The complex-valued functions $\left(\tilde{E}_{x}, \tilde{E}_{y}, \tilde{E}_{z}, \tilde{B}_{x}, \tilde{B}_{y}, \tilde{B}_{z}\right)$ represent the stationary solution of the electromagnetic field in a given structure; $E_{x}(x, y, z, t)=\Re\left[\tilde{E}_{x}(x, y, z) e^{i \omega_{0} t}\right]$ etc. The components of the electromagnetic field under the integrals are taken at the electron position parametrized by $z$ :

$$
(x, y, z)=\left[x_{1}+x_{1}^{\prime}\left(z-z_{1}\right), y_{1}+y_{1}^{\prime}\left(z-z_{1}\right), z\right] .
$$

It is assumed here that the motion of the electrons is piecewise linear, with straight line trajectory within one unit cell, from $z_{1}$ to $z_{2}=z_{1}+\lambda_{p}$; although the electron accumulates momentum during its flight through the cell, in calculation the accumulated momentum is added only at the exit of the cell; this is equivalent to the Euler method of solving differential equations (a first-order RungeKutta method). This method is numerically less efficient than the conventional fourth-order Runge-Kutta algorithm, but the formulas are simpler, easier to derive, analyze, expand in series, and this facilitates elementary physical insight.

The validity of the Euler approximation was checked for the calculations of Secs. IV and V by subdividing the unit cell into four subcells, and calculating the unit cell transfer function as a composition $\mathcal{R}=\mathcal{R}_{4} \mathcal{R}_{3} \mathcal{R}_{2} \mathcal{R}_{1}$, where $\mathcal{R}_{1}$ is the transfer function from $z$ to $z+\frac{1}{4} \lambda_{\mathrm{p}}$, etc. Such refinement did not influence the $\left(x_{2}, x_{2}^{\prime}\right)$ plots in Figs. 4 and 7(c). On the other hand, the refinement did quantitatively influence the calculation shown in Fig. 7(b), where the accelerator segment consisted of only two elementary cells. In this case the calculation converged for $n \approx 50$ subdivision segments, and this large number of segments was used to produce Fig. 7(b).

Equations (A1) contain small dimensionless parameters $x^{\prime}, y^{\prime}, \delta, \Delta p_{i} / p_{0}$. In textbooks on conventional accelerators such equations are usually expanded in Taylor series and higher order terms are dropped [5]. For the purposes of this paper Taylor expansion of Eq. (A1) would not be productive. Note that linearization of the transfer function is not possible, as discussed in Sec. IV.

\section{APPENDIX B: DERIVATION OF THE TRANSFER FUNCTION EQUATIONS}

Assuming the electron trajectory is linear within the unit cell (or its subset, see previous section), as the electron travels from $z_{1}$ to $z_{2}$, its transverse position $x$ increases from $x_{1}$ to $x_{2}$, with $x_{2}=x_{1}+\Delta x=x_{1}+\frac{\Delta x}{\Delta z} \Delta z=$ $x_{1}+x_{1}^{\prime}\left(z_{2}-z_{1}\right)$. Similarly, $y_{2}=y_{1}+y_{1}^{\prime}\left(z_{2}-z_{1}\right)$.

$C$ is the cosine of the deflection of electron trajectory from the $\hat{z}$ direction, $C=\hat{z} \cdot \hat{v}_{1}=(0,0,1) \cdot \frac{\left(v_{1 x}, v_{1 y}, v_{1 z}\right)}{v_{1}}$, where $\vec{v}_{1}$ is the velocity of the electron at the entrance of the cell. It follows that $C=\frac{v_{1 z}}{v_{1}}=$ $\frac{v_{1 z} d t}{v_{1} d t}=\frac{d z}{\sqrt{d x^{2}+d y^{2}+d z^{2}}}=\frac{1}{\sqrt{(d x / d z)^{2}+(d y / d z)^{2}+(d z / d z)^{2}}}=\frac{1}{\sqrt{x_{1}^{\prime 2}+y_{1}^{\prime 2}+1}}$.

Momentum and velocity at the entrance of the cell are related by $p_{1}=\frac{1}{\sqrt{1-\beta_{1}^{2}}} m \beta_{1} c$, or $\beta_{1}=\frac{p_{1}}{\sqrt{p_{1}^{2}+m^{2} c^{2}}}$, or, using the definition of $\delta$ [Eq. (10)], $\beta_{1}=\frac{p_{0}\left(1+\delta_{1}\right)}{\sqrt{p_{0}^{2}\left(1+\delta_{1}\right)^{2}+m^{2} c^{2}}}$. The $z$ component of the relative velocity is $\beta_{1 z}=\frac{\beta_{1 z}}{\beta_{1}} \beta_{1}=$ $C \frac{p_{0}\left(1+\delta_{1}\right)}{\sqrt{p_{0}^{2}\left(1+\delta_{1}\right)^{2}+m^{2} c^{2}}}$.

The slope at the exit of the cell is $x_{2}^{\prime}=\frac{d x}{d z}=\frac{p_{2 x}}{p_{2 z}}=\frac{p_{1 x}+\Delta p_{x}}{p_{1 z}+\Delta p_{z}}=\frac{p_{1 x} / p_{1 z}+\Delta p_{x} / p_{1 z}}{p_{1 z} / p_{1 z}+\Delta p_{z} / p_{1 z}}=\frac{x_{1}^{\prime}+\Delta p_{x} / p_{1 z}}{1+\Delta p_{z} / p_{1 z}}=\frac{x_{1}^{\prime}+\Delta p_{x} / C p_{1}}{1+\Delta p_{z} / C p_{1}}=$ $\frac{x_{1}^{\prime}+\Delta p_{x} / C p_{0}\left(1+\delta_{1}\right)}{1+\Delta p_{z} / C p_{0}\left(1+\delta_{1}\right)}$. The expression for $y_{2}^{\prime}$ is analogous.

The momentum at the exit of the cell is $p_{2}=\sqrt{\left(p_{1 x}+\Delta p_{x}\right)^{2}+\left(p_{1 y}+\Delta p_{y}\right)^{2}+\left(p_{1 z}+\Delta p_{z}\right)^{2}}=$ $p_{1 z} \sqrt{\left(\frac{p_{1 x}}{p_{1 z}}+\frac{\Delta p_{x}}{p_{1 z}}\right)^{2}+\left(\frac{p_{1 y}}{p_{1 z}}+\frac{\Delta p_{y}}{p_{1 z}}\right)^{2}+\left(\frac{p_{1 z}}{p_{1 z}}+\frac{\Delta p_{z}}{p_{1 z}}\right)^{2}}=C p_{1} \sqrt{\left(x_{1}^{\prime}+\frac{\Delta p_{x}}{C p_{1}}\right)^{2}+\left(y_{1}^{\prime}+\frac{\Delta p_{y}}{C p_{1}}\right)^{2}+\left(1+\frac{\Delta p_{z}}{C p_{1}}\right)^{2}}=C p_{0}\left(1+\delta_{1}\right) \times$ $\sqrt{\left(x_{1}^{\prime}+\frac{\Delta p_{x}}{C p_{0}\left(1+\delta_{1}\right)}\right)^{2}+\left(y_{1}^{\prime}+\frac{\Delta p_{y}}{C p_{0}\left(1+\delta_{1}\right)}\right)^{2}+\left(1+\frac{\Delta p_{z}}{C p_{0}\left(1+\delta_{1}\right)}\right)^{2}}$, so the relative momentum deviation is by definition (10) $\delta_{2}=p_{2} / p_{0}-1=C\left(1+\delta_{1}\right) \sqrt{\left(x_{1}^{\prime}+\frac{\Delta p_{x}}{C p_{0}\left(1+\delta_{1}\right)}\right)^{2}+\left(y_{1}^{\prime}+\frac{\Delta p_{y}}{C p_{0}\left(1+\delta_{1}\right)}\right)^{2}+\left(1+\frac{\Delta p_{z}}{C p_{0}\left(1+\delta_{1}\right)}\right)^{2}}-1$.

The phase increases from $\Phi_{1}=\omega_{0} t_{1}$ to $\Phi_{2}=\omega_{0} t_{2}$, and $\Phi_{2}=\Phi_{1}+\omega_{0} \Delta t=\Phi_{1}+\omega_{0} \frac{\Delta z}{\beta_{z} c}=\Phi_{1}+\frac{k_{0}\left(z_{2}-z_{1}\right)}{\beta_{z}}$.

During its flight through the cell the electron receives momentum $\left(\Delta p_{x}, \Delta p_{y}, \Delta p_{z}\right)$ from the electromagnetic field, where $\Delta p_{x}=\int F_{x} d t=\int \frac{F_{x}}{d z / d t} d z=\int \frac{F_{x}}{c \beta_{z}} d z=\frac{(-e)}{c} \int \frac{1}{\beta_{z}}\left(E_{x}+\beta_{y} c B_{z}-\beta_{z} c B_{y}\right) d z=\frac{(-e)}{c} \int\left(\frac{1}{\beta_{z}} E_{x}+y_{1}^{\prime} c B_{z}-c B_{y}\right) d z$. The electromagnetic field components under the integral are taken at the electron location, parametrized by $z: E_{x}=E_{x}(x, y, z, t)=$ $\Re\left\{\tilde{E}_{x}(x, y, z) \exp \left[i \omega_{0} t\right]\right\}=\Re\left\{\tilde{E}_{x}[x(z), y(z), z] \exp \left[i \omega_{0} t(z)\right]\right\}=\Re\left\{\tilde{E}_{x}\left[x_{1}+x_{1}^{\prime}\left(z-z_{1}\right), y_{1}+y_{1}^{\prime}\left(z-z_{1}\right), z\right] \exp \left[i\left(\Phi_{1}+\frac{k_{0}\left(z-z_{1}\right)}{\beta_{x}}\right)\right]\right\}$, and similarly for $E_{y}$ and $E_{z}$. The real-part operator $\Re$ is additive and in the expression for $\Delta p_{x}$ can act as the final operation: $\Delta p_{x}=\Re\left\{\frac{(-e)}{c} \int\left(\frac{1}{\beta_{z}} \tilde{E}_{x}+y_{1}^{\prime} c \tilde{B}_{z}-c \tilde{B}_{y}\right) \exp \left[i\left(\Phi_{1}+\frac{k_{0}\left(z-z_{1}\right)}{\beta_{x}}\right)\right] d z\right\}$. The derivation of expressions for $\Delta p_{y}$ and $\Delta p_{z}$ is similar. 


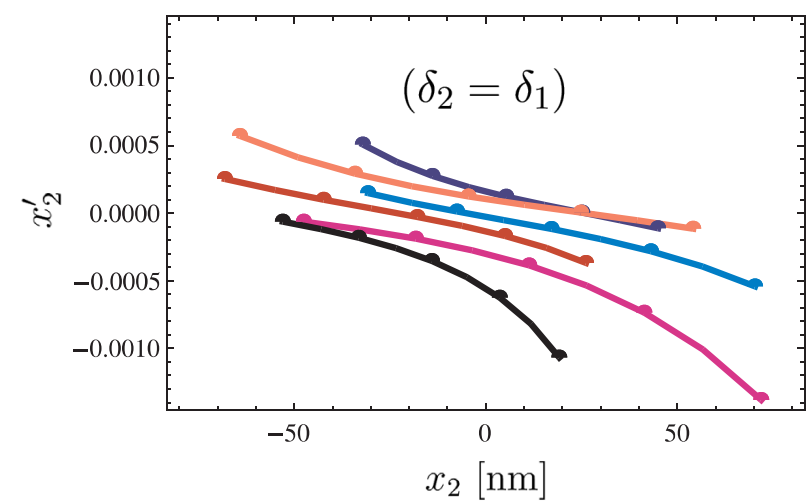

FIG. 10. The focusing properties of the $\mathcal{O}_{5+1 / 2} \mathcal{R}^{8} \mathcal{O}_{5+1 / 2} \mathcal{R}^{8}$ segment calculated using "spoiled" $\left(\delta_{2}=\delta_{1}\right)$ transfer function [compare with the correct result in Fig. 7(c)].

\section{APPENDIX C: ARE VARIATIONS IN $\delta$ SIGNIFICANT FOR FOCUSING?}

In Sec. $\mathrm{V}$ the forces on an electron traversing a $\mathcal{O}_{m+1 / 2} \mathcal{R}^{n} \mathcal{O}_{m+1 / 2} \mathcal{R}^{n}$ structure are discussed, and it is shown that the overall effect is focusing. The argument, based on $\left(x, x^{\prime}\right)$ plots for a single cell, is purely geometric, assuming $\delta=0$ and thus neglecting the "chromatic effects." However, the calculations leading to Fig. 7 are exact in the sense that full transfer function is used [Eqs. (A1)], so in the calculation $\delta$ is nonzero (except the entrance of the cell). Is focusing modified by chromatic effects $(\delta \neq 0)$ ? To answer this question, let us "spoil" the transformation (A1) by assuming $\delta_{2}=\delta_{1}$ instead of Eq. (A1f). This means that now $\delta$ is forced to remain constant, equal to the initial zero value, and that the phase $\Phi$ advances in each elementary cell by exactly $2 \pi$. The result is shown in Fig. 10. The structure still has focusing properties, but the result is significantly different than for the correct transformation, and the average focusing power decreases by a factor of $\sim 2$. So the "geometric argument," while essentially correct, does not capture all focusing factors, and chromatic effects are also important.

\section{APPENDIX D: PONDEROMOTIVE FOCUSING AND PONDEROMOTIVE FORCE- QUANTITATIVE ANALYSIS}

Suppose a particle is subject to an oscillating force $\vec{F}=\vec{F}_{0} \cos \omega t$, whose amplitude $\vec{F}_{0}$ varies spatially on length scales larger than the amplitude of the $\omega$ oscillation of the particle. Under these circumstances an effective, average force on the particle arises, called the ponderomotive force (see e.g. $[23,24])$ :

$$
\vec{F}_{\mathrm{p}} \sim-\frac{1}{\omega^{2}} \nabla\left(\left|\vec{F}_{0}\right|^{2}\right) .
$$

For a high-energy particle traversing a FODO-like DLA structure described in Sec. V, the transverse defecting force (a)

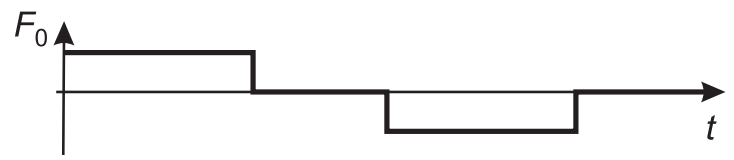

(b)

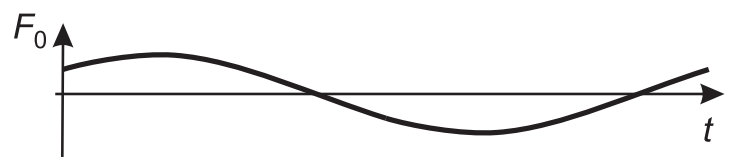

FIG. 11. (a) Time dependence of the focusing force in a FODOlike DLA cell (Fig. 6). (b) Harmonic oscillation leading to classical ponderomotive force.

is a function of transverse position $(x, y)$ and oscillates with frequency $\omega^{\prime}$ given by Eq. (15), causing small-amplitude electron oscillation in the $(x, y)$ plane, so the basic requirements for ponderomotive force are satisfied. The distinction between a single "FODO" cell and repeated "FODOFODO..." structure does not affect the physical focusing mechanism and should not affect the terminology. There is however one significant difference between the classical ponderomotive force and the present situation: the oscillation of the focusing force is not harmonic, as shown in Fig. 11. This sheds doubt on the applicability of Eq. (D1) to the present situation. The derivation of this equation $[23,24]$ should be reconsidered, allowing for nonharmonic force oscillations, which is beyond the scope of this paper. Nevertheless, let us numerically check three features of ponderomotive focusing occurring in the $\mathcal{O}_{5+1 / 2} \mathcal{R}^{8} \mathcal{O}_{5+1 / 2} \mathcal{R}^{8}$ structure, and compare them with Eq. (D1).

(1) Let us reduce the amplitude of force oscillation $F_{0}$ by half by reducing driving laser amplitude $E_{0}$ (see Fig. 3) by half. The calculation yields the result that the average focusing power of the structure decreases by a factor of 4.2 , signifying the decrease of the ponderomotive focusing force by the same factor. This result is close to the value of 4 expected from Eq. (D1).

(2) Let us shorten the structure approximately by half: $\mathcal{O}_{3+1 / 2} \mathcal{R}^{4} \mathcal{O}_{3+1 / 2} \mathcal{R}^{4}$. This increases the oscillation frequency by a factor of 2 . The result is that the average focusing power decreases by a factor of 7.5. This is actually closer to $2^{3}$ than to the value $2^{2}$ expected from Eq. (D1) and questions the applicability of this equation to nonharmonic oscillating forces.

(3) Let us, for the structure $\mathcal{O}_{5+1 / 2} \mathcal{R}^{8} \mathcal{O}_{5+1 / 2} \mathcal{R}^{8}$, calculate the gradient $\nabla\left(F_{0}^{2}\right)=\frac{\partial}{\partial x}\left(F_{0}^{2}\right)$. The force amplitude $F_{0}$ is equal to the average force exerted on the electron traversing an elementary DLA cell

$$
F_{0}=\left\langle F_{x}\right\rangle \sim \Delta p_{x} \sim \Delta x^{\prime}=x_{2}^{\prime}-x_{1}^{\prime},
$$




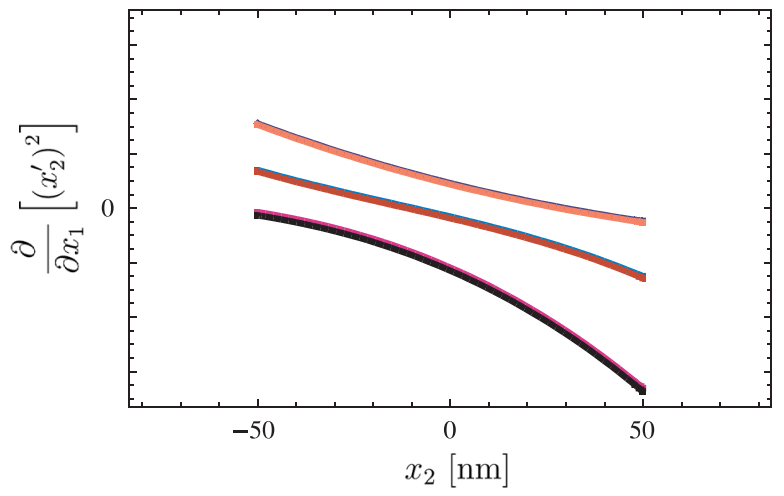

FIG. 12. Equation (D4), based on the transfer properties of an elementary DLA cell, plotted for the same six phases as in Fig. 7.

where an approximate form $x_{2}^{\prime}=x_{1}^{\prime}+\Delta p_{x} / p_{0}$ of Eq. (A1b) was used. For simplification, a transfer function for $x_{1}^{\prime}=0$ is considered here:

$$
\left(x_{1}, 0,0,0, \Phi_{1}, 0\right) \stackrel{\mathcal{R}}{\rightarrow}\left(x_{2}, x_{2}^{\prime}, 0,0, \Phi_{2}, \delta_{2}\right),
$$

so the force amplitude $F_{0}$ is simply proportional to $x_{2}^{\prime}$ for an elementary cell, where $x_{2}^{\prime}$ is a function of six parameters: $x_{2}^{\prime}=x_{2}^{\prime}\left(x_{1}, 0,0,0, \Phi_{1}, 0\right)$. The final equation for the gradient, neglecting multiplicative constants, is

$$
\nabla\left(F_{0}^{2}\right) \sim \frac{\partial}{\partial x_{1}}\left[\left(x_{2}^{\prime}\right)^{2}\right] .
$$

This functional dependence is plotted in Fig. 12. If Eq. (D1) was strictly valid, the plots in Figs 7(c) and 12 should be the same up to a multiplicative constant. While both plots indicate focusing, there are quantitative differences, so Eq. (D1) is not strictly valid for DLA ponderomotive focusing force.

There could be one more reason for the inaccuracy of Eq. (D1) in the present situation. Perhaps the transverse oscillation amplitude of the electron is too large. This hypothesis may be verified in future work.

[1] R. Joel England et al., Dielectric laser accelerators, Rev. Mod. Phys. 86, 1337 (2014).

[2] K. P. Wootton, J. McNeur, and K. J. Leedle, Dielectric laser accelerators: Designs, experiments, and applications, Rev. Accel. Sci. Techol. 09, 105 (2016).

[3] J. Breuer and P. Hommelhoff, Laser-Based Acceleration of Nonrelativistic Electrons at a Dielectric Structure, Phys. Rev. Lett. 111, 134803 (2013).

[4] K. L. Brown and R. V. Servranckx, Technical Report No. SLAC-PUB-3381, Stanford University, 1984.

[5] K. Wille, The Physics of Particle Accelerators. An Introduction (Oxford University Press, Oxford, UK, 2000).
[6] H. Wiedemann, Particle Accelerator Physics (Springer, New York, 2015).

[7] Course materials of U.S. Particle Accelerator School, http://uspas.fnal.gov/materials/, 2017.

[8] J. D. Joannopoulos, S. G. Johnson, J. N. Winn, and R. D. Meade, Photonic Crystals: Molding the Flow of Light, 2nd ed. (Princeton University Press, Princeton, NJ, 2008).

[9] K. Soong, Particle accelerator on a wafer: Demonstration of electron acceleration and diagnostics with microstructures, Ph.D. thesis, Stanford University, 2014.

[10] E. Hemsing, G. Stupakov, D. Xiang, and A. Zholents, Beam by design: Laser manipulation of electrons in modern accelerators, Rev. Mod. Phys. 86, 897 (2014).

[11] G. Brooker, Modern Classical Optics (Oxford University Press, Oxford, UK, 2007).

[12] A. Szczepkowicz, Guided-mode resonance, resonant grating thickness, and finite-size effects in dielectric laser acceleration structures, Appl. Opt. 55, 2634 (2016).

[13] J. Breuer, J. McNeur, and P. Hommelhoff, Dielectric laser acceleration of electrons in the vicinity of single and double grating structures-Theory and simulations, J. Phys. B 47, 234004 (2014).

[14] J. Hoffrogge, J. P. Stein, M. Krüger, M. Förster, J. Hammer, D. Ehberger, P. Baum, and P. Hommelhoff, Tip-based source of femtosecond electron pulses at $30 \mathrm{keV}$, J. Appl. Phys. 115, 094506 (2014).

[15] K. J. Leedle, A. Ceballos, H. Dend, O. Solgaard, R. Fabian Pease, R. L. Byer, and J. S. Harris, Dielectric laser acceleration of sub-100 keV electrons with silicon dual-pillar grating structures, Opt. Lett. 40, 4344 (2015).

[16] A. Ody, P. Musumeci, J. Maxson, D. Cesar, R. J. England, and K. P. Wootton, Flat electron beam sources for DLA accelerators, Nucl. Instrum. Methods Phys. Res., Sect. A 865, 75 (2017).

[17] E. Prat, S. Bettoni, M. Calvi, M. Dehler, F. Frei, P. Hommelhoff, M. Kozak, J. McNeur, C. Ozkan Loch, S. Reiche, A. Romann, and R. Ischebeck, Outline of a dielectric laser acceleration experiment at SwissFEL, Nucl. Instrum. Methods Phys. Res., Sect. A 865, 87 (2017).

[18] T. Plettner, R. L. Byer, C. McGuinness, and P. Hommelhoff, Photonic-based laser driven electron beam deflection and focusing structures, Phys. Rev. ST Accel. Beams 12, 101302 (2009).

[19] K. Soong, R. L. Byer, E. R. Colby, R. J. England, and E. A. Peralta, Grating-based deflecting, focusing, and diagnostic dielectric laser accelerator structures, AIP Conf. Proc. 1507, 516 (2012).

[20] K. P. Wootton, D. B. Cesar, C. Lee, I. V. Makasyuk, J. Maxson, P. Musumeci, and R. J. England, Dielectric laser acceleration and focusing using short-pulse lasers with an arbitrary laser phase distribution, AIP Conf. Proc. 1812, 060001 (2017).

[21] J. McNeur, M. Kozák, N. Schönenberger, K. J. Leedle, H. Deng, A. Ceballos, H. Hoogland, A. Ruehl, I. Hartl, R. Holzwarth, O. Solgaard, J. S. Harris, R. L. Byer, and P. Hommelhoff, Elements of a dielectric laser accelerator, arXiv:1604.07684.

[22] B. Naranjo, A. Valloni, S. Putterman, and J. B. Rosenzweig, Stable Charged-Particle Acceleration and Focusing in a 
Laser Accelerator Using Spatial Harmonics, Phys. Rev. Lett. 109, 164803 (2012).

[23] P. Mulser, Physical picture of parametric phenomena and ponderomotive effects in solids, in Nonlinear Optics in Solids: Proceedings of the International Summer School, Aalborg, Denmark, 1989, edited by O. Keller (Springer, Berlin, 1990), pp. 112-135.

[24] A. Macchi, A Superintense Laser-Plasma Interaction Theory Primer (Springer, New York, 2013).
[25] S.C. Hartman and J. B. Rosenzweig, Ponderomotive focusing in axisymmetric rf linacs, Phys. Rev. E 47, 2031 (1993).

[26] T. P Wangler, RF Linear Accelerators (Wiley, New York, 2008).

[27] D. A. Swenson, Alternating phase focused linacs, Part. Accel. 7, 61 (1976).

[28] E. F. F. Chladni, Entdeckungen über die Theorie des Klanges (Weidmanns Erben und Reich, Leipzig, 1787). 